\title{
Stability of a coupling technique for partitioned solvers in FSI applications
}

\author{
Joris Degroote $^{\mathrm{a}}$, Peter Bruggeman ${ }^{\mathrm{b}}$, Robby Haelterman ${ }^{\mathrm{c}}$, \\ Jan Vierendeels $*, a$

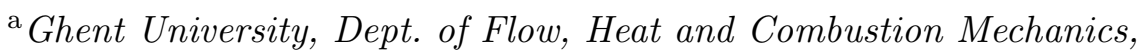 \\ St.-Pietersnieuwstraat 41, B-9000 Ghent, Belgium \\ ${ }^{\mathrm{b}}$ Ghent University, Dept. of Applied Physics, Jozef Plateaustraat 22, B-9000 \\ Ghent, Belgium \\ ${ }^{\mathrm{c}}$ Royal Military Academy, Dept. of Mathematics (MWMW), Renaissancelaan 30, \\ B-1000 Brussels, Belgium
}

\begin{abstract}
This paper focuses on the stability of the coupling iterations in the partitioned approach to fluid-structure interaction. Previous research has shown that the number of coupling iterations increases when the time step decreases or when the structure becomes more flexible which is explained here by Fourier error analysis of the unsteady, incompressible flow in an elastic tube. Substituting a linearized model of the structural solver into the flow solver makes the coupling more stable but is impracticable if the flow solver is a black box. Therefore the coupling iterations are stabilized by coupling with reduced-order models and Aitken underrelaxation.
\end{abstract}

Key words: elastic tube, artery, stability, fluid-structure interaction, Fourier error analysis, reduced-order model, black box

PACS: 65M12, 65Z05, 76D45, 76T10 


\section{Introduction}

Recently fluid-structure interaction has received a lot of attention in several domains. In aeronautics [1] and turbomachinery [2], fluid-structure interaction is used for flutter analysis. Parachute dynamics is another interesting field as large deformations appear [3]. Biomedical applications (e.g. heart valves [4], arteries $[5,6])$ are challenging as the interaction between the fluid and structure is strong due to the flexible structure and the comparable density of the fluid and the structure.

Fluid-structure interaction and other coupled problems can be dealt with using a monolithic or a partitioned approach. In the monolithic approach, both the flow and structure problem are solved with a single code [7-11]. This avoids the coupling iterations to find the coupled solution in the partitioned approach where the flow and structure are solved with separate codes. On the other hand, the monolithic approach nowadays requires the use of a dedicated code [7], whereas the partitioned approach allows the reuse of existing codes as long as the solvers for flow and structure are treated as black boxes. Only partitioned approaches to fluid-structure interaction are considered here.

Several coupling schemes for the partitioned approach have been devised. In a staggered scheme, the flow and structure problem are solved sequentially and no coupling iterations are performed within a time step [12-14]. This scheme

* Corresponding author.

Email address: Jan.Vierendeels@UGent. be (Jan Vierendeels).

$U R L$ : www.floheacom. ugent. be (Jan Vierendeels). 
is only stable in cases with weak interaction between the fluid and the solid, like aeroelastic applications.

Before introducing coupling schemes that do require coupling iterations, the flow solver $(\mathcal{F}$ or $f)$ and the structural solver $(\mathcal{S}$ or $s)$ are written in fixed point formulation

$$
\begin{aligned}
& Y=\mathcal{F}(X) \\
& X=\mathcal{S}(Y)
\end{aligned}
$$

and root-finding formulation

$$
\begin{array}{r}
f(X, Y)=\mathcal{F}(X)-Y=0 \\
s(X, Y)=\mathcal{S}(Y)-X=0
\end{array}
$$

with $X$ the position of the fluid-structure boundary and $Y$ the fluid load on it. The fluid-structure interaction problem in the latter formulation can be solved with Newton-Raphson iterations $[15,16]$. If the linear systems are solved with a direct method, this approach requires knowledge of the Jacobian of the equations (2), which can be very time consuming or difficult to calculate for black box solvers. Therefore also quasi-Newton methods with approximate Jacobians are used [17-19].

In the fixed point formulation, the coupled solution can be found with coupling iterations between the flow and structure problem. However, this is prone to instability when the density ratio of fluid and structure increases and when the structure becomes more flexible $[20,21]$. In previous research $[5,22,23]$ with a very flexible, light or zero-mass structure and an incompressible fluid, it is shown that the number of coupling iterations increases when the time step decreases. So unlike many other situations, decreasing the time step does not 
facilitate the solution of the problem [24,25]. It is important to understand this tendency in order to improve coupling techniques for the partitioned solution of coupled problems.

There exist several techniques to stabilize the coupling iterations between a black box fluid and structural solver, like Aitken's method [26], which uses a variable underrelaxation of the fixed point iterations. A scheme to couple two black box solvers by making linear reduced-order models (ROMs) of those solvers has been introduced by Vierendeels et al. [5]. Aitken underrelaxation and coupling with reduced-order models will be discussed further in this paper.

Notwithstanding the focus of this paper on coupling techniques which do not require modification of the partitioned solvers, a lot of insight can be gained by substituting a linearized model of the structure into the flow solver. The substitution terms in the flow equations can be activated or deactivated, so coupling schemes ranging from monolithic to completely partitioned can be analyzed.

To enable both analytical and numerical analysis, a one-dimensional problem is studied namely the unsteady flow in an elastic tube. This is a simplified model of the pulsating blood flow in an artery for which two-dimensional simulations have been presented in previous work [5]. Although a one-dimensional model does not accurately represent the complex phenomena in an artery, its behaviour from a fluid-structure point of view is very similar. A onedimensional model is outstandingly suited to analyze the effect of the time step, space step and elasticity of the structure on the number of coupling iterations. Fourier error analysis is used to investigate the stability of the coupling iterations between flow and structure for several coupling schemes. 
The remainder of this paper is organized as follows. A detailed description of the flow and structure problem is given in section 2 and a simple fixed point iterative coupling scheme is introduced in section 3. The Fourier error analysis of this scheme follows in section 4 and the results of the error analysis are verified in section 5 by means of simulations with a linear structure model. In section 6 the coupling scheme with reduced-order models and Aitken underrelaxation are outlined and in section 7 the performance of coupling with reduced-order models is compared with fixed point iterative coupling with and without Aitken underrelaxation using a non-linear structure model.

\section{Problem description}

\subsection{Analytical description}

The unsteady flow in a tube is analyzed with a one-dimensional model, shown in Figure 1. The fluid is incompressible and inviscid and gravity is neglected. The governing equations are the conservation of mass and momentum which can be written in conservative form as

$$
\begin{gathered}
\frac{\partial a}{\partial t}+\frac{\partial a u}{\partial x}=0 \\
\frac{\partial a u}{\partial t}+\frac{\partial a u^{2}}{\partial x}+\frac{1}{\rho}\left(\frac{\partial a \mathcal{P}}{\partial x}-\mathcal{P} \frac{\partial a}{\partial x}\right)=0
\end{gathered}
$$

with $a$ the cross sectional area of the tube, $u$ the velocity along the axis of the tube and $\frac{\partial}{\partial t}$ the time derivative. $x$ is the spatial coordinate, $\rho$ is the density of the fluid and $\mathcal{P}$ the pressure.

The behaviour of the elastic tube wall is described with a Hookean constitutive relation. The structure contains no mass, as the inertia of the tube wall is 
neglected with regards to that of the fluid. An axisymmetrical model is used in coordinate system $(x, r, \varphi)$, with $r$ the inner radius of the tube and $\varphi$ the angle in the cross sectional plane as shown in Figure 1. The stress in the tube wall in circumferential direction $\sigma_{\varphi \varphi}$ is approximated as

$$
\sigma_{\varphi \varphi}=E \frac{r-r_{0}}{r_{0}}+\sigma_{0}
$$

with $E$ Young's modulus and $r_{0}$ the radius for which $\sigma_{\varphi \varphi}=\sigma_{0}$. Other stress components are neglected. This model allows only radial motion of the tube wall and the force balance on the fluid-structure interface is

$$
\mathcal{P} r=\sigma_{\varphi \varphi} h
$$

with $h$ the thickness of the tube wall.

By substituting the constitutive equation (4) and the kinematic pressure $p=$ $\mathcal{P} / \rho$ in (5), the following relation holds

$$
r p=\frac{E h}{\rho r_{0}}\left(r-r_{0}\right)+r_{0} p_{0}
$$

with $\mathcal{P}_{0} r_{0}=\sigma_{0} h$. This can be rewritten as

$$
a=a_{0}\left(\frac{p_{0}-2 c_{m k}^{2}}{p-2 c_{m k}^{2}}\right)^{2}
$$

by using $a=\pi r^{2}$ and by introducing the Moens-Korteweg wave speed

$$
c_{m k}=\sqrt{\frac{E h}{2 \rho r_{0}}} .
$$

The tube wall thus has a constitutive law of the form $a=a(p)$, with the cross sectional area only a function of the local pressure, so (3) can be rewritten in 
the following form

$$
\begin{aligned}
& \frac{\partial p}{\partial t}+u \frac{\partial p}{\partial x}+c^{2} \frac{\partial u}{\partial x}=0 \\
& \frac{\partial u}{\partial t}+\frac{\partial p}{\partial x}+u \frac{\partial u}{\partial x}=0
\end{aligned}
$$

The wave speed $c$ is defined as

$$
c^{2}=\frac{a}{\frac{\mathrm{d} a}{\mathrm{~d} p}}
$$

and it can easily be verified that the system (9) has 2 eigenvalues: $\lambda_{1,2}=u \pm c$. Even though the fluid is incompressible, the wave speed is finite due to the fluid-structure interaction. With (7), the wave speed becomes

$$
c^{2}=c_{m k}^{2}-\frac{p}{2}
$$

The velocity is imposed at the inlet of the tube and a non-reflecting boundary

$$
\frac{\mathrm{d} u}{\mathrm{~d} t}=\frac{1}{c} \frac{\mathrm{d} p}{\mathrm{~d} t}
$$

is prescribed at the outlet.

\subsection{Discretization}

The straight tube with circular cross section and length $L$ is discretized with a one-dimensional mesh with $N$ cells of length $\Delta x$, as indicated on Figure 1 . The fluid velocity and pressure are stored in the cell centres. Central discretization is used for all terms in the continuity and momentum equation, except for the convective term in the momentum equation which is discretized with a firstorder upwind scheme. The time discretization scheme is backward Euler and the time step is indicated with $\Delta t$. The conservation of mass and momentum 
in a control volume around cell centre $i$ is expressed by the following system of equations

$$
\begin{aligned}
& \frac{\Delta x}{\Delta t}\left(a_{i}-a_{i}^{n}\right)+u_{i+1 / 2} a_{i+1 / 2}-u_{i-1 / 2} a_{i-1 / 2}-\alpha\left(p_{i+1}-2 p_{i}+p_{i-1}\right)=0 \\
& \begin{aligned}
\frac{\Delta x}{\Delta t}\left(u_{i} a_{i}-u_{i}^{n} a_{i}^{n}\right)+u_{i} u_{i+1 / 2} a_{i+1 / 2}-u_{i-1} u_{i-1 / 2} a_{i-1 / 2} \\
+\frac{1}{2}\left(a_{i+1 / 2}\left(p_{i+1}-p_{i}\right)+a_{i-1 / 2}\left(p_{i}-p_{i-1}\right)\right)=0
\end{aligned}
\end{aligned}
$$

for $u_{i} \geq 0$. The subscripts $i, i+1$ and $i-1$ indicate the cell centres $(i=$ $1, \ldots, N)$ and the subscript $i \pm 1 / 2$ signifies the values calculated at the cell interfaces, $u_{i-1 / 2}=\left(u_{i-1}+u_{i}\right) / 2$ and $u_{i+1 / 2}=\left(u_{i}+u_{i+1}\right) / 2$. The superscript $n$ denotes the previous time level, the notation $n+1$ for the new time level is omitted. A pressure stabilization term with coefficient $\alpha=a_{0} /\left(u_{0}+\Delta x / \Delta t\right)$ has been added in the continuity equation to prohibit pressure wiggles due to central discretization of the pressure in the momentum equation, with $u_{0}$ the initial flow velocity.

The pressure at the inlet and the velocity at the outlet are linearly extrapolated

$$
\begin{aligned}
p_{\text {in }} & =2 p_{1}-p_{2} \\
u_{\text {out }} & =2 u_{N}-u_{N-1}
\end{aligned}
$$

and the pressure-outlet condition (12) is discretized as

$$
p_{\text {out }}=2\left[c_{m k}^{2}-\left(\sqrt{c_{m k}^{2}-\frac{p_{\text {out }}^{n}}{2}}-\frac{u_{\text {out }}-u_{\text {out }}^{n}}{4}\right)^{2}\right],
$$

taking into account the variation of $c$ with $p$ given by equation (11) when integrating from time level $n$ to $n+1$.

The geometrical discretization of the elastic problem is identical to that of the flow problem to avoid errors in the data transfer between the flow and the 
structure. Equation (7) is discretized as

$$
a_{i}=a_{0}\left(\frac{p_{0}-2 c_{m k}^{2}}{p_{i}-2 c_{m k}^{2}}\right)^{2}
$$

and linearization of this equation around state 0 results in

$$
a_{i}-a_{0}=\frac{a_{0}}{c_{0}^{2}}\left(p_{i}-p_{0}\right)
$$

\section{Iterative coupling scheme}

The one-dimensional problem has been introduced to investigate the behaviour of coupling schemes with fixed point iterations. In this section, a simple fixed point iterative scheme between the flow problem and the structure problem is given and subsequently modified by inserting the linearized structure model in the flow problem. This section and the following one elucidate why the coupling scheme with reduced-order models (section 6) performs well and what the remaining problem is.

A simple fixed point iterative scheme to calculate the fully coupled solution for a time step can be implemented as follows, with superscript $k$ indicating the coupling iteration:

(1) Solve equations (13) for the new velocity and pressure, $u_{i}^{k+1}$ and $p_{i}^{k+1}$, with a fixed geometry $a_{i}^{k}(i=1, \ldots, N)$.

(2) Calculate the new geometry $a_{i}^{k+1}$ from the linear equation (17) or the non-linear equation (16) given the previously calculated pressure $p_{i}^{k+1}$ $(i=1, \ldots, N)$.

(3) Increase $k$ and return to step 1 until convergence is obtained. 
The position of the fluid-structure interface at the beginning of the first coupling iteration is calculated with a second order extrapolation from the previous time steps. This coupling scheme does not perform well in most cases with strong interaction between fluid and structure. If this iteration scheme converges, often a large number of coupling iterations is needed.

The performance of the fixed point iterative coupling scheme is significantly improved by using the linearized elastic equation (17) to add information on the elastic wall in the flow equations. Irrespective of this linear substitution, the structure equation itself can be linear or non-linear. The fixed point iterative scheme is thus modified to the following one, resulting from the substitution of $a$ by $a^{k}+\gamma_{j} a_{0} / c_{0}^{2}\left(p^{k+1}-p^{k}\right)$ in equations (13). Quadratic terms in $p$ are omitted and the substitution terms can be activated or deactivated by setting the parameters $\gamma_{j}(j=1, \ldots, 4)$ to 1 or to 0 in order to identify the coupling terms that prevent the instability of the coupling iterations.

(1) Solve

$$
\begin{aligned}
& \frac{\Delta x}{\Delta t}\left(a_{i}^{k}-a_{i}^{n}\right)+\gamma_{1} \frac{\Delta x}{\Delta t} \frac{a_{0}}{c_{0}^{2}}\left(p_{i}^{k+1}-p_{i}^{k}\right)+u_{i+1 / 2}^{k+1} a_{i+1 / 2}^{k}-u_{i-1 / 2}^{k+1} a_{i-1 / 2}^{k} \\
& +\gamma_{2}\left(u_{i+1 / 2}^{k+1} \frac{a_{0}}{c_{0}^{2}}\left(p_{i+1 / 2}^{k+1}-p_{i+1 / 2}^{k}\right)-u_{i-1 / 2}^{k+1} \frac{a_{0}}{c_{0}^{2}}\left(p_{i-1 / 2}^{k+1}-p_{i-1 / 2}^{k}\right)\right) \\
& -\alpha\left(p_{i+1}^{k+1}-2 p_{i}^{k+1}+p_{i-1}^{k+1}\right)=0 \\
& \frac{\Delta x}{\Delta t}\left(u_{i}^{k+1} a_{i}^{k}-u_{i}^{n} a_{i}^{n}\right)+\gamma_{3} u_{i}^{k+1} \frac{\Delta x}{\Delta t} \frac{a_{0}}{c_{0}^{2}}\left(p_{i}^{k+1}-p_{i}^{k}\right) \\
& +u_{i}^{k+1} u_{i+1 / 2}^{k+1} a_{i+1 / 2}^{k}-u_{i-1}^{k+1} u_{i-1 / 2}^{k+1} a_{i-1 / 2}^{k} \\
& +\gamma_{4}\left(u_{i}^{k+1} u_{i+1 / 2}^{k+1} \frac{a_{0}}{c_{0}^{2}}\left(p_{i+1 / 2}^{k+1}-p_{i+1 / 2}^{k}\right)-u_{i-1}^{k+1} u_{i-1 / 2}^{k+1} \frac{a_{0}}{c_{0}^{2}}\left(p_{i-1 / 2}^{k+1}-p_{i-1 / 2}^{k}\right)\right) \\
& +\frac{1}{2}\left(a_{i+1 / 2}^{k}\left(p_{i+1}^{k+1}-p_{i}^{k+1}\right)+a_{i-1 / 2}^{k}\left(p_{i}^{k+1}-p_{i-1}^{k+1}\right)\right)=0
\end{aligned}
$$


for the new velocity and pressure, $u_{i}^{k+1}$ and $p_{i}^{k+1}$, with a fixed geometry $a_{i}^{k}(i=1, \ldots, N)$.

(2) Calculate the new geometry $a_{i}^{k+1}$ with the linear relation

$$
a_{i}^{k+1}=a_{0}+\frac{a_{0}}{c_{0}^{2}}\left(p_{i}^{k+1}-p_{0}\right)
$$

or with the non-linear relation

$$
a_{i}^{k+1}=a_{0}\left(\frac{p_{0}-2 c_{m k}^{2}}{p_{i}^{k+1}-2 c_{m k}^{2}}\right)^{2}
$$

given the previously calculated pressure $p_{i}^{k+1}(i=1, \ldots, N)$.

(3) Increase $k$ and return to step 1 until convergence is obtained.

Although this paper focuses on coupling black box solvers, this is useful because various coupling schemes ranging from monolithic to partitioned can be studied. If all the coupling terms $\left(\gamma_{j}=1, j=1, \ldots, 4\right)$ and the linear elastic equation (19) are used, then the modified fixed point iterative scheme is in reality a monolithic code and it should converge in one coupling iteration. This property and the importance of the different coupling terms are investigated in the following section.

\section{Fourier error analysis}

The stability of the fixed point iterative coupling scheme with extra coupling terms in the flow equations is now investigated with Fourier error analysis without taking the boundary conditions into account. The velocity, pressure and cross sectional area at the new time level in equations (18) and (19) are written as the sum of the coupled solution and the remaining error, indicated with a tilde 


$$
\begin{aligned}
& u_{i}^{k}=u_{i}+\tilde{u}_{i}^{k} \\
& p_{i}^{k}=p_{i}+\tilde{p}_{i}^{k} \\
& a_{i}^{k}=a_{i}+\tilde{a}_{i}^{k}
\end{aligned}
$$

for $i=1, \ldots, N$. The coupled solutions at both time level $n$ and $n+1$ are decomposed as $u_{0}, p_{0}$ and $a_{0}$ and a small perturbation. Subsequently, the equations are linearized by neglecting all non-linear combinations of the error terms and the perturbations. Considering that these equations linearized around $u_{0}$, $p_{0}$ and $a_{0}$ are also satisfied by the coupled solutions, all perturbations cancel out, resulting in the following set of linearized equations for the error terms.

$$
\begin{aligned}
& \frac{\Delta x}{\Delta t} \tilde{a}_{i}^{k}+\gamma_{1} \frac{\Delta x}{\Delta t} \frac{a_{0}}{c_{0}^{2}}\left(\tilde{p}_{i}^{k+1}-\tilde{p}_{i}^{k}\right)+u_{0}\left(\tilde{a}_{i+1 / 2}^{k}-\tilde{a}_{i-1 / 2}^{k}\right)+a_{0}\left(\tilde{u}_{i+1 / 2}^{k+1}-\tilde{u}_{i-1 / 2}^{k+1}\right) \\
& +\gamma_{2} u_{0} \frac{a_{0}}{c_{0}^{2}}\left(\tilde{p}_{i+1 / 2}^{k+1}-\tilde{p}_{i+1 / 2}^{k}-\tilde{p}_{i-1 / 2}^{k+1}+\tilde{p}_{i-1 / 2}^{k}\right)-\alpha\left(\tilde{p}_{i+1}^{k+1}-2 \tilde{p}_{i}^{k+1}+\tilde{p}_{i-1}^{k+1}\right)=0
\end{aligned}
$$

$$
\begin{gathered}
\frac{\Delta x}{\Delta t}\left(u_{0} \tilde{a}_{i}^{k}+\tilde{u}_{i}^{k+1} a_{0}\right)+\gamma_{3} u_{0} \frac{\Delta x}{\Delta t} \frac{a_{0}}{c_{0}^{2}}\left(\tilde{p}_{i}^{k+1}-\tilde{p}_{i}^{k}\right)+u_{0}^{2}\left(\tilde{a}_{i+1 / 2}^{k}-\tilde{a}_{i-1 / 2}^{k}\right) \\
+u_{0} a_{0}\left(\tilde{u}_{i+1 / 2}^{k+1}+\tilde{u}_{i}^{k+1}-\tilde{u}_{i-1 / 2}^{k+1}-\tilde{u}_{i-1}^{k+1}\right)+\gamma_{4} u_{0}^{2} \frac{a_{0}}{c_{0}^{2}}\left(\tilde{p}_{i+1 / 2}^{k+1}-\tilde{p}_{i+1 / 2}^{k}-\tilde{p}_{i-1 / 2}^{k+1}+\tilde{p}_{i-1 / 2}^{k}\right) \\
+\frac{1}{2} a_{0}\left(\tilde{p}_{i+1}^{k+1}-\tilde{p}_{i-1}^{k+1}\right)=0 \\
\quad \text { (22b) } \\
\tilde{a}_{i}^{k+1}=\frac{a_{0}}{c_{0}^{2}} \tilde{p}_{i}^{k+1}
\end{gathered}
$$

The error terms are expanded as the sum of $N$ Fourier modes, giving

$$
\tilde{u}_{i}^{k}=\frac{1}{N} \sum_{l=0}^{N-1} \tilde{u}_{l}^{k} e^{\jmath \omega_{l} i \Delta x}
$$

with $\jmath=\sqrt{-1}$ and the spatial pulsation $\omega_{l}=\frac{2 \pi l}{L}$. As equations (22) are linear in the error terms, every Fourier mode can be studied separately so the 
following substitution is performed

$$
\tilde{u}_{i}^{k} \rightarrow \tilde{u}_{l}^{k} e^{\jmath \omega_{l} i \Delta x}
$$

and $\tilde{p}_{i}^{k}$ and $\tilde{a}_{i}^{k}$ are substituted analogously.

The resulting equations are non-dimensionalized by dividing them by $a_{0} c_{0}$, $a_{0} c_{0}^{2}$ and $a_{0}$, respectively. The non-dimensional parameters and variables are defined as

$$
\begin{array}{llrl}
\frac{\tilde{a}_{l}^{k}}{a_{0}}=A_{l}^{k} & \frac{\tilde{p}_{l}^{k}}{c_{0}^{2}}=P_{l}^{k} & \frac{\alpha c_{0}}{a_{0}}=\beta \\
\frac{\tilde{u}_{l}^{k}}{c_{0}}=U_{l}^{k} & \frac{u_{0}}{c_{0}}=U_{0} & \frac{\Delta x / \Delta t}{c_{0}}=D_{0}
\end{array}
$$

and $\beta=1 /\left(U_{0}+D_{0}\right)$. With $\theta_{l}=\omega_{l} \Delta x=\frac{2 \pi l}{N}$, the following non-dimensional equations are obtained.

$$
\begin{gathered}
D_{0} A_{l}^{k}+\gamma_{1} D_{0}\left(P_{l}^{k+1}-P_{l}^{k}\right)+U_{0} A_{l}^{k} \jmath \sin \left(\theta_{l}\right)+U_{l}^{k+1} \jmath \sin \left(\theta_{l}\right) \\
+\gamma_{2} U_{0}\left(P_{l}^{k+1}-P_{l}^{k}\right) \jmath \sin \left(\theta_{l}\right)-2 \beta P_{l}^{k+1}\left(\cos \left(\theta_{l}\right)-1\right)=0 \\
D_{0} U_{0} A_{l}^{k}+D_{0} U_{l}^{k+1}+\gamma_{3} D_{0} U_{0}\left(P_{l}^{k+1}-P_{l}^{k}\right)+U_{0}^{2} A_{l}^{k} \jmath \sin \left(\theta_{l}\right) \\
+U_{0} U_{l}^{k+1}\left(\jmath \sin \left(\theta_{l}\right)+1-e^{-\jmath \theta_{l}}\right)+\gamma_{4} U_{0}^{2}\left(P_{l}^{k+1}-P_{l}^{k}\right) \jmath \sin \left(\theta_{l}\right) \\
+P_{l}^{k+1} \jmath \sin \left(\theta_{l}\right)=0 \\
A_{l}^{k+1}=P_{l}^{k+1}
\end{gathered}
$$

Every coupling iteration, the component with spatial frequency $\omega_{l}$ of the error on the interface's position and on the fluid load is amplified by

$$
\begin{aligned}
& \frac{A_{l}^{k+1}}{A_{l}^{k}}=\frac{P_{l}^{k+1}}{P_{l}^{k}} \\
& \quad=1+\frac{-U_{0}^{2}\left(1-e^{-\jmath \theta_{l}}\right) \jmath \sin \theta_{l}-b_{1} D_{0}-b_{2}}{\gamma_{1} b_{1} D_{0}+\gamma_{2} b_{1} U_{0} \jmath \sin \theta_{l}-\gamma_{3} D_{0} U_{0} \jmath \sin \theta_{l}+\gamma_{4} U_{0}^{2} \sin ^{2} \theta_{l}+b_{2}}
\end{aligned}
$$


with

$$
\begin{gathered}
b_{1}=D_{0}+U_{0}\left(\jmath \sin \theta_{l}+1-e^{-\jmath \theta_{l}}\right) \\
b_{2}=\sin ^{2} \theta_{l}-2 b_{1} \beta\left(\cos \theta_{l}-1\right)
\end{gathered}
$$

which is a function of $\theta_{l}$ and the parameters $U_{0}$ and $D_{0}$ only. Only the amplification of $A_{l}$ and $P_{l}$ is studied as only these values are exchanged between the solvers during the coupling iterations. The iteration scheme will be stable for given values of $U_{0}$ and $D_{0}$ if the error amplification factor $\mu_{l}$ is less than or equal to one for all $\theta_{l}$.

$$
\mu_{l}=\left|\frac{A_{l}^{k+1}}{A_{l}^{k}}\right|=\left|\frac{P_{l}^{k+1}}{P_{l}^{k}}\right| \leq 1
$$

To study the relation between the stability of the coupling iterations and the structural stiffness, the time step $\Delta t$ and the space step $\Delta x$, the following combinations of $U_{0}, D_{0}$ and $N$ are defined.

$$
\kappa=\frac{1}{U_{0}}=\frac{\sqrt{\frac{E h}{2 \rho r_{0}}-\frac{p_{0}}{2}}}{u_{0}} \quad \tau=\frac{U_{0}}{D_{0} N}=\frac{u_{0} \Delta t}{L}
$$

In this work, mainly the elasticity coefficient $E$ and the time step $\Delta t$ are varied so $\kappa$ represents the dimensionless structural stiffness and $\tau$ the dimensionless time step. The effect of the reference flow velocity $u_{0}$ can be seen by modifying $\kappa$ and $\tau$ such that $\kappa \tau$ remains constant. The radius $r_{0}$ and the length $L$ of the tube can be influenced through $\kappa$ and $\tau$, respectively. Approximate values of $\kappa$ and $\tau$ for a simulation of a large human artery are $\kappa \approx 100$ and $\tau \approx 0.01$.

The error amplitude reduction of the fixed point iterative scheme is now studied for different values of the parameters $\kappa$ and $\tau$ and with the most significant combinations of the coupling terms (indicated by the values of $\gamma_{1}, \gamma_{2}, \gamma_{3}$ and $\left.\gamma_{4}\right)$. The following conclusions can be drawn from the study of the error am- 
plification factor.

If $\gamma_{1}=\gamma_{2}=\gamma_{3}=\gamma_{4}=1$, the coupling is monolithic and thus $\mu_{l}=0$ for all $\theta_{l}$, irrespective of $\kappa, \tau$ and $N$. Only one coupling iteration will be required.

If $\gamma_{1}=\gamma_{2}=\gamma_{3}=\gamma_{4}=0$, the coupling is completely partitioned and equations (28) simplify to

$$
\mu_{l}=\frac{1}{\kappa^{2}}\left|\begin{array}{c}
(\tau N)^{3}\left(1-e^{-\jmath \theta_{l}}\right) \jmath \sin \theta_{l}+(\tau N)^{2}\left[\jmath \sin \theta_{l}+\left(1-e^{-\jmath \theta_{l}}\right)\left(1+\jmath \sin \theta_{l}\right)\right] \\
+\tau N\left(\jmath \sin \theta_{l}+2-e^{-\jmath \theta_{l}}\right)+1 \\
(\tau N)^{3}\left[\sin ^{2} \theta_{l}-2\left(\jmath \sin \theta_{l}+1-e^{-\jmath \theta_{l}}\right)\left(\cos \theta_{l}-1\right)\right] \\
+(\tau N)^{2}\left[\sin ^{2} \theta_{l}-2\left(\cos \theta_{l}-1\right)\right]
\end{array}\right|
$$

Figure 2 and Figure 3 show that the error amplification increases for decreasing

$\theta$. The lowest frequency components are the most unstable ones during fixed point iterative coupling without extra coupling terms. From equation (31), it is clear that the mode with $\theta_{l}=0$ is always unstable if $\gamma_{1}=0$. As can be seen in Figure 2 and equation (31), the instability grows quadratically when the dimensionless stiffness $\kappa$ decreases. For $u_{0}=0, \kappa$ is infinite and thus $\mu_{l}=0$ for all $\theta_{l}$. Figure 3 illustrates that a smaller dimensionless time step $\tau$ makes higher spatial frequencies unstable if no coupling terms are used. The instability thus grows when the time step or the structural stiffness decreases without coupling terms. The effect of $\kappa$ is generally greater than that of $\tau N$ and $\kappa$ determines the vertical position of the curve while $\tau N$ modifies both its shape and position. $\tau N$ influences the stability significantly in Figure 3(b) where $\kappa$ is small $(\kappa=10)$ but not in Figure 3(a). An increase of $N$ by some 
factor has the same effect on the curve as an increase of $\tau$ with the same factor, namely the maximal $\theta_{l}$ for which $\mu_{l}>1$ decreases, which is a stabilizing effect. On the other hand, the number of Fourier modes rises for increasing $N$ as the difference $2 \pi / N$ between the $\theta_{l}$ decreases. This is a destabilizing effect and together these influences cause a variation of the number of unstable frequencies with $N$ as shown in Figure 4. The number of unstable frequencies thus only varies significantly with $N$ for a flexible structure and a small time step. For $\kappa=10$ and $\tau=0.001$, all frequencies are unstable as long as $N \leq 51$.

In Figure 5(a) the reduction of the error amplification due to the coupling term premultiplied with $\gamma_{1}$ can be seen. When $\gamma_{1}=1, \kappa$ influences both the shape and the vertical position of the curve. $\tau N$ is an order of magnitude smaller than in Figure 2(a) and yet the error amplification factor is smaller than one for all $\theta_{l}$. This coupling term is often referred to as the artificial compressibility term [27-29]. Normally it is used in stepping schemes for the incompressible flow equations. Here it is used to stabilize the coupling between the incompressible flow equations and an elastic wall. Figure 5(b) shows that with artificial compressibility $\left(\gamma_{1}=1\right)$, the error amplification remains nearly unchanged when $\tau N$ is decreased, as opposed to Figure 3 where a smaller $\tau N$ increased the instability.

In Figure 6(a), two configurations are shown for which the term premultiplied with $\gamma_{1}$ is not sufficient to stabilize the fixed point iterations. This situation can appear when $\kappa<1$ while $\tau>1$. This extreme case means that the convective speed is larger than the critical speed in an iteration where the solution is sought for a time step which is too large to follow the convective phenomena accurately. As can be seen in Figure 6(b), the only way to stabilize the previously mentioned extreme case is to add the convective coupling term 
premultiplied by $\gamma_{2}$ in the continuity equation. It can also be noticed that the coupling term premultiplied by $\gamma_{3}$ cannot stabilize this case.

Since the addition of the artificial compressibility term $\left(\gamma_{1}\right)$ in the continuity equation ensures the linear stability in all practical cases, only this term will be considered in the following.

\section{Results with linear structure}

Since the flow equations are non-linear and the previous Fourier error analysis is linear, a one-dimensional numerical experiment is performed to investigate the influence of the non-linearity in the flow equations on the stability. The structure is modeled by the linear equation (17). The real error amplitude reduction can be observed during the experiment by following the L1-norm of the change of cross sectional area and of the change of fluid load during the coupling iterations for the solution of a time step.

At the inlet, the velocity is imposed as

$$
U_{0}+\frac{U_{0}}{100} \sin ^{2}(\pi n \tau / T)
$$

with the period $T=1$. This perturbation of the inlet velocity only results in a limited non-linearity of the flow problem. The pressure is initially zero in all nodes and the velocity has a uniform initial value $U_{0}$. Due to the discretization and boundary conditions, the flow velocity has to be lower than the wave speed at all times. One period of the inlet velocity's variation is simulated and it is verified that the difference between the resulting wave speed and the wave speed from a monolithic simulation is smaller than $0.001 \%$, which means that 
a sufficiently converged solution is obtained in each time step.

Although the Fourier error analysis indicates that a spatially uniform error component is unstable, it is possible to simulate a very stiff tube $(\kappa=1000)$ with fixed point iterations without coupling terms in the flow solver. This is because the boundary conditions could not be be taken into account in the Fourier analysis. By imposing the velocity at the inlet of the tube, the velocityerror with $\theta=0$ is fixed and by using an outlet condition $\Delta u \sim \Delta p$ and a wall model $a=a(p)$, this also determines the components of error on $p$ and $a$ with $\theta=0$.

Figure 7 shows the convergence behaviour of the L1-norm of the change in cross sectional area and change in fluid load during the coupling iterations in a representative time step for three configurations with different $\kappa$ and constant $\tau=0.01$ and $N=100$. A representative time step required approximately the average number of coupling iterations. The coupling iterations in a time step have converged when both the normalized change in cross sectional area and the change in fluid load are smaller than the convergence criterion $1 \mathrm{E}-$ 7. When $\kappa=1000$, the simple fixed point iteration scheme with $\gamma_{1}=0$ converges in 7.88 coupling iterations on average. It does not converge for a more flexible structure. If $\gamma_{1}=1,4.50$ coupling iterations are required on average for the same case. As the time step decreases or the structure becomes more flexible, the artificial compressibility coupling term $\left(\gamma_{1}=1\right)$ is needed to obtain convergence.

The number of coupling iterations per time step averaged over one period of the inlet boundary condition (32) is given in Table 1 for different values of $\kappa, \tau$ and $N$. As expected, the number of coupling iterations increases as 
$\kappa$ decreases. Without the artificial compressibility $\left(\gamma_{1}=0\right)$, the number of coupling iterations increases fast with decreasing $\tau$. On the contrary, a decrease in $\tau$ causes only a minor increase of the number of coupling iterations when the artificial compressibility term is used $\left(\gamma_{1}=1\right)$. The influence of $N$ is small, except for $\kappa=1000$ and $\tau=0.001$ with $\gamma_{1}=0$, which is the only configuration from Figure 4 that can be simulated. Although Figure 4 predicts that this configuration has only one unstable frequency which will be stabilized by the boundary conditions, there is a significant variation of the number of coupling iterations with $N$. Stability of the simulation due to the boundary conditions is no guarantee for fast convergence because there are a lot of stable, lightly damped error modes. Taking into account a limited non-linearity of the flow equations thus does not alter the conclusions drawn from the Fourier error analysis.

\section{Coupling scheme with reduced-order models and Aitken under- relaxation}

Adding extra coupling terms in the flow equations might be a good approach for a one-dimensional case. It is however more involved in two or three dimensions, especially when the user does not want to modify the flow code a lot. Unlike the fixed point iterative coupling scheme with extra coupling terms in the flow equations, the coupling scheme with reduced-order models treats the flow solver and structural solver as black boxes, which is a significant advantage for more complex problems.

The fixed point iterative coupling scheme without coupling terms is an explicit coupling technique within the coupling iterations of a time step. This means 
that the flow problem is solved for the fluid load on the fluid-structure interface without taking in account the deformation of the geometry due to the change in fluid load. Analogously for the structure problem. A monolithic code solves the flow and structure problem simultaneously which is an implicit solution strategy.

The details of the coupling algorithm of Vierendeels et al. [5] are given in appendix A for completeness, here a less formal explanation follows. For the one-dimensional tube, the position $X$ of the fluid-structure interface and the fluid load $Y$, both defined in equation (1), are given by

$$
\begin{aligned}
& X=\left[\begin{array}{llll}
a_{1} & a_{2} & \ldots & a_{N}
\end{array}\right]^{\mathrm{T}} \\
& Y=\left[\begin{array}{llll}
p_{1} & p_{2} & \ldots & p_{N}
\end{array}\right]^{\mathrm{T}} .
\end{aligned}
$$

The input and output of the solvers do not represent the entire domain but are limited to the fluid-structure interface.

In every coupling iteration of a time step, the structure and flow problem are solved successively. Every time the flow problem has been solved, an input and the corresponding output of the flow solver is known. Using those inputs and outputs, a reduced-order model for the flow solver is generated. As additional inputs and outputs are calculated during the coupling iterations, this reducedorder model becomes more accurate. The same technique is used to generate a reduced-order model for the structural solver.

Every coupling iteration consists of the steps indicated in Figure 8. In the initialization, the position of the fluid-structure interface at the beginning of the first coupling iteration is calculated with a second order extrapola- 
tion from the previous time steps and two coupling iterations are performed, with underrelaxation in the second one. After the initialization, the position of the fluid-structure interface is calculated as the coupled solution of the reduced-order models and then the corresponding fluid load on the interface is calculated with the black box flow solver. Afterwards, the reduced-order model of the flow solver is updated with the latest input and output of the flow solver. Subsequently an analogous procedure is followed for the structural solver. Coupling iterations are performed upon convergence. Any linear combination of the displacements and the changes in fluid load that are known by the reduced-order models is coupled implicitly as the coupled solution of the reduced-order models is calculated. The coupling is explicit for the displacements and the changes in fluid load that cannot be written as a linear combination of those in the reduced-order models.

The Fourier error analysis of the fixed point iterative coupling without coupling terms showed that the variations with a low spatial frequency cause the instability of the explicit coupling of the black box solvers and that the instability increases with decreasing time step or structural stiffness. As not all variations are unstable, it is sufficient to couple the unstable frequency components implicitly. As the most unstable variations will appear first in the inputs and outputs of the solvers during the coupling iterations, they will be included in the reduced-order models from the beginning of the coupling iterations and thus they will be coupled implicitly rapidly.

The calculation of the coupled solution of the reduced-order models in equations (43) and (44) requires the solution of a linear system with a dense matrix. However, the number of rows and columns of this dense matrix is equal to the number of degrees-of-freedom of the fluid-structure interface which is much 
smaller than the number of degrees-of-freedom in the entire fluid-structural domain for two- and three-dimensional simulations. Moreover, matrix-free iterative linear solvers can be used to solve these linear systems in a fast and efficient way. The computational cost of the reduced-order models is thus small with respect to the computational cost of the flow solver and the structural solver.

For Aitken underrelaxation, the flow solver and the structural solver are used consecutively within a coupling iteration to calculate a preliminary position of the fluid-structure interface, indicated with a tilde, as

$$
\tilde{X}^{k+1}=\mathcal{S}\left(\mathcal{F}\left(X^{k}\right)\right)
$$

The corresponding residual array is given by $R^{k+1}=\mathcal{S}\left(\mathcal{F}\left(X^{k}\right)\right)-X^{k}=\tilde{X}^{k+1}-$ $X^{k}$ and the Aitken underrelaxation factor is subsequently calculated as

$$
\omega^{k}=-\omega^{k-1} \frac{\left(R^{k}\right)^{\mathrm{T}}\left(R^{k+1}-R^{k}\right)}{\left(R^{k+1}-R^{k}\right)^{\mathrm{T}}\left(R^{k+1}-R^{k}\right)}
$$

The position of the fluid-structure interface then becomes

$$
X^{k+1}=\omega^{k} \tilde{X}^{k+1}+\left(1-\omega^{k}\right) X^{k} .
$$

For more details about this method, the reader is referred to $[24,25]$.

\section{Results with non-linear structure}

The simulations from section 5 are repeated, but now the structure is modeled with the non-linear constitutive equation (16) and the amplitude of the varying velocity component in equation $(32)$ is $U_{0} / 10$ which results in a larger non-linearity of the flow problem. The convergence behaviour of fixed point 
iterative coupling with artificial compressibility is very similar to Figure 7 so it is not shown. Figure 9 depicts the convergence behaviour for coupling with reduced-order models during the coupling iterations in a representative time step for three different values of $\kappa$. In Figure 10, the same is shown for Aitken underrelaxation.

The reduced-order models can only be constructed when at least two iterations have been performed because otherwise no differences between the inputs and outputs of the solvers can be calculated. Consequently, underrelaxation has to be used in the second coupling iteration (step (5) in appendix A) and as such the change in area is significantly smaller than in the first iteration as can be seen in Figure 9. After the second coupling iteration, the reduced-order models can be used and the convergence is monotonic. To avoid that a time step is considered as converged before the onset of the monotonic convergence of the coupling iterations, at least 3 coupling iterations have to be performed. While the convergence of coupling with reduced-order models is monotonic as soon as the reduced-order models are available, the convergence of Aitken underrelaxation (Figure 10) never becomes monotonic.

The number of coupling iterations per time step averaged over one period of the inlet boundary condition (32) is given in Table 2. As explained above, it increases for decreasing $\kappa$ for all coupling techniques. For $\kappa=100$ and $\tau=0.1$, the three techniques require on average an equal number of coupling iterations, but as the time step is decreased the number of coupling iterations increases slowly for the fixed point iterative coupling with artificial compressibility and for reduced-order model coupling and much faster for Aitken underrelaxation. The effect of $N$ on the number of coupling iterations is small for each coupling technique. The results of the Fourier error analysis on the fixed point iterative 
coupling are thus also valid for coupling with reduced-order models and Aitken underrelaxation.

It is important to observe that Aitken underrelaxation requires twice as many coupling iterations as reduced-order model coupling for $\kappa=10$ and $\tau<0.1$. As has been shown, the number of unstable frequencies increases rapidly with decreasing $\tau$ for a very flexible structure. Coupling with reduced-order models decomposes the change of the position of the interface and the load on the interface and handles every component separately. Aitken underrelaxation, on the other hand, uses the same underrelaxation factor for the entire displacement in a coupling iteration which becomes increasingly difficult when more modes become unstable.

Axisymmetrical 2D simulations of a flexible tube using coupling with reducedorder models have been presented in [5]. In those simulations, only 5 coupling iterations were required for $\kappa \approx 1000$ and $\tau=0.001$, so even fewer than the approximately 7 coupling iterations mentioned in Table 2 for the same values of $\kappa$ and $\tau$ because in this work the inertia of the structure has been neglected. The absence of inertia in the structure is a more severe test for the coupling algorithms because the displacement of the structure subjected to a pressure that has a certain error with respect to the solution of the time step will be larger.

As the length $L$ or the radius $r_{0}$ of the tube is increased, respectively $\tau$ or $\kappa$ decreases, both yielding more unstable fixed point iterations. In [20], it is shown that the largest eigenvalue of the so-called added mass operator increases as the tube becomes longer and more narrow. The maximal underrelaxation factor for which fixed point Dirichlet-Neumann iterations are stable is then 
calculated as a combination of the maximal eigenvalue of the added mass operator, the mass of the wall and a stiffness parameter. This underrelaxation factor increases when the radius increases, which is due to the mass of the wall. When the inertia of the wall is chosen two orders of magnitude smaller, the underrelaxation factor decreases for increasing radius meaning that the simulation becomes more unstable as observed in this paper without taking into account the inertia of the wall. Causin et al. determine for which underrelaxation factor fixed point iterations are stable, while in this paper it has been shown analytically which frequency components become unstable and how the number of unstable Fourier modes in the coupling iterations varies with $\kappa, \tau$ and $N$ and that this is clearly correlated with the number of coupling iterations. Also, the effect of $N$ has been shown both analytically and numerically.

In section 5 and section 7 , it is demonstrated that when the time step is decreased in a partitioned fluid-structure interaction simulation with black box solvers where the mass of the structure is negligible and the fluid is incompressible, the number of coupling iterations in a time step will increase, which is a highly unwanted behaviour that has also been observed in two-dimensional simulations $[5,20,30]$. For the coupling with reduced-order models, it should be possible to alter this behaviour by reusing data from the previous time level(s) in the reduced-order models or by extrapolating reduced-order models from the previous time level(s) to the new time level, which is currently investigated by the authors. 


\section{Conclusions}

Components of the solution with a low spatial frequency destabilize explicit fixed point coupling iterations between a black box flow solver and a structural solver for the unsteady flow in an elastic tube. The number of cells has a small influence on the number of coupling iterations but when the time step is decreased or the structure becomes more flexible, the number of coupling iterations increases. The instability can be reduced by substituting a linearized model for the structure into the flow solver. This is however not possible for black box solvers. Coupling with reduced-order models and Aitken underrelaxation are valuable coupling techniques that do not require modifications of the solvers. The performance of the coupling with reduced-order models is superior to that of Aitken underrelaxation. Unstable components appear during the first coupling iterations so they are included in the reduced-order models and thus they are coupled implicitly in the subsequent coupling iterations. Techniques that treat the solvers as black boxes and that do not require more coupling iterations when the time step decreases have to be developed.

\section{Acknowledgments}

The authors gratefully acknowledge the funding of Joris Degroote and Peter Bruggeman by a Ph.D. fellowship of the Research Foundation - Flanders (FWO). Joris Degroote acknowledges a grant for a long stay abroad at the Massachusetts Institute of Technology (MIT) of the Research Foundation

- Flanders. Jan Vierendeels acknowledges the funding by Research Project G027508 of the Research Foundation - Flanders and the BOF Association. 


\section{A Detailed description of the coupling scheme with reduced-order models}

The coupling scheme of Vierendeels et al. [5] for a flow solver $Y=\mathcal{F}(X)$ and a structural solver $X=\mathcal{S}(Y)$ can be written as follows. How a reducedorder model is generated, is explained later. The subscripts $f$ and $s$ denote fluid respectively structure and a superscript $k$ or $k^{\prime}$ indicates the coupling iteration whereas $n$ is the time level.

(1) Calculate an initial position for the interface $X_{f}$ at time level $n+1$ from the state known at the previous time levels, e.g. $X_{f}^{1}=\frac{5}{2} X^{n}-2 X^{n-1}+$ $\frac{1}{2} X^{n-2}$.

(2) Calculate the corresponding fluid load $Y_{f}^{1}=\mathcal{F}\left(X_{f}^{1}\right)$ with the flow solver.

(3) Estimate the fluid load that has to be applied at the interface by the structural solver, e.g. $Y_{s}^{1}=Y_{f}^{1}$.

(4) Calculate the position of the interface with the structural solver $X_{s}^{1}=$ $\mathcal{S}\left(Y_{s}^{1}\right)$

(5) Estimate the position of the interface that has to be applied by the flow solver, e.g. $X_{f}^{2}=X_{f}^{1}+\omega\left(X_{s}^{1}-X_{f}^{1}\right)$, with $\omega$ an underrelaxation factor.

(6) Calculate the corresponding fluid load with the flow solver $Y_{f}^{2}=\mathcal{F}\left(X_{f}^{2}\right)$.

(7) With $k=2$, update the reduced-order model for the flow problem.

(8) Estimate the fluid load that has to be applied at the interface by the structural solver, e.g. $Y_{s}^{2}=Y_{f}^{2}$.

(9) Calculate the position of the interface with the structural solver $X_{s}^{2}=$ $\mathcal{S}\left(Y_{s}^{2}\right)$

(10) With $k^{\prime}=2$, update the reduced-order model for the structural problem.

(11) Compute $X_{f}^{k+1}=\hat{X}$ as the coupled solution of the reduced-order models. 
(12) Compute the corresponding fluid load with the flow solver $Y_{f}^{k+1}=\mathcal{F}\left(X_{f}^{k+1}\right)$.

(13) Update the reduced-order model for the flow problem with $k=k+1$.

(14) Compute $Y_{s}^{k^{\prime}+1}=\hat{Y}$ as the coupled solution of the reduced-order models.

(15) Compute the corresponding position with the structural solver $X_{s}^{k^{\prime}+1}=$ $\mathcal{S}\left(Y_{s}^{k^{\prime}+1}\right)$.

(16) Exit the loop if convergence is achieved.

(17) Update the reduced-order model for the structural solver with $k^{\prime}=k^{\prime}+1$.

(18) Go to step 11.

The reduced-order models for the solvers can be built up during the coupling iterations of each time step. Below it is demonstrated how a reduced-order model for the flow solver is obtained. Assume that the flow solver has been called $k$ times during the coupling iterations, then the flow solver has computed the fluid loads $Y_{f}^{i}(i=1, \ldots, k)$ that correspond with the interface positions $X_{f}^{i}(i=1, \ldots, k)$. Using this information, a matrix

$$
V=\left[\begin{array}{lll}
\Delta X_{f}^{1} \Delta X_{f}^{2} \ldots \Delta X_{f}^{k-1}
\end{array}\right]
$$

with displacement modes $\Delta X_{f}^{j}=X_{f}^{j}-X_{f}^{k}(j=1, \ldots, k-1)$ and a matrix

$$
W=\left[\begin{array}{llll}
\Delta Y_{f}^{1} & \Delta Y_{f}^{2} \ldots \Delta Y_{f}^{k-1}
\end{array}\right]
$$

with the corresponding changes in fluid load distributions $\Delta Y_{f}^{j}=Y_{f}^{j}-Y_{f}^{k}$ $(j=1, \ldots, k-1)$ are constructed. An arbitrary displacement $\Delta X_{f}$ can be approximated by a linear combination of the known displacement modes.

$$
\Delta X_{f} \approx V \alpha
$$

with

$$
\alpha=\left[\begin{array}{ccc}
\alpha_{1} \ldots \alpha_{k-1} & \\
]^{\mathrm{T}}
\end{array}\right.
$$


The corresponding change in fluid load distribution $\Delta Y_{f}$ can thus be approximated by $\Delta Y_{f}=W \alpha$. Minimization of $\left\|\Delta X_{f}-V \alpha\right\|_{2}$ results in a least squares problem with solution $\alpha=\left(V^{\mathrm{T}} V\right)^{-1} V^{\mathrm{T}} \Delta X_{f}$ so that $\Delta Y_{f}=\hat{F}_{X}^{k} \Delta X_{f}$ with

$$
\hat{F}_{X}^{k}=W\left(V^{\mathrm{T}} V\right)^{-1} V^{\mathrm{T}}
$$

where a hat indicates an approximation. The resulting reduced-order model for the flow problem is

$$
\hat{Y}=Y_{f}^{k}+\hat{F}_{X}^{k}\left(\hat{X}-X_{f}^{k}\right)
$$

In a similar way, the reduced-order model for the structural solver can be built. Assume there were $k^{\prime}$ calls of the structural solver, then the reducedorder model can be written as

$$
\hat{X}=X_{s}^{k^{\prime}}+\hat{S}_{Y}^{k^{\prime}}\left(\hat{Y}-Y_{s}^{k^{\prime}}\right)
$$

The reduced-order models for the fluid and structure can be solved for $\hat{X}$

$$
\hat{X}=\left(I-\hat{S}_{Y}^{k^{\prime}} \hat{F}_{X}^{k}\right)^{-1}\left[X_{s}^{k^{\prime}}+\hat{S}_{Y}^{k^{\prime}}\left(Y_{f}^{k}-Y_{s}^{k^{\prime}}-\hat{F}_{X}^{k} X_{f}^{k}\right)\right]
$$

or for $\hat{Y}$

$$
\hat{Y}=\left(I-\hat{F}_{X}^{k} \hat{S}_{Y}^{k^{\prime}}\right)^{-1}\left[Y_{f}^{k}+\hat{F}_{X}^{k}\left(X_{s}^{k^{\prime}}-X_{f}^{k}-\hat{S}_{Y}^{k^{\prime}} Y_{s}^{k^{\prime}}\right)\right] .
$$

\section{References}

[1] Dowell E, Thomas J, Hall K. Transonic limit cycle oscillation analysis using reduced order aerodynamic models. Journal of Fluids and Structures 2004; 19(1):17-27. 
[2] Willcox K, Paduano J, Peraire J. Low order aerodynamic models for aeroelastic control of turbomachines. In: 40th AIAA ASME ASCE AHS ASC Structures, Structural Dynamics and Materials Conference. St Louis, MO: AIAA ASME ASCE AHS ASC, 1999 1-11.

[3] Stein K, Tezduyar T, Kumar V, Sathe S, Benney R, Charles R. Numerical simulation of soft landing for clusters of cargo parachutes. In: Niettaanmäki P, Rossi T, Majava K, Pieronneau O, editors, European congres on computational methods in applied sciences and engineering ECCOMAS 2004. Jyväskylä, Finland, 2004 1-14.

[4] dos Santos N, Gerbeau JF, Bourgat JF. Partitioned fsi strategy for simulations of a thin elastic valve. In: Wesseling P, Oñate E, Périaux J, editors, European Conference on Computational Fluid Dynamics ECCOMAS CFD 2006. Delft, The Netherlands: ECCOMAS, 2006 1-10.

[5] Vierendeels J, Lanoye L, Degroote J, Verdonck P. Implicit coupling of partitioned fluid-structure interaction problems with reduced order models. Computers and Structures 2007; 85:970-976.

[6] Scotti C, Finol E. Compliant biomechanics of abdominal aortic aneurysms: A fluid-structure interaction study. Computers and Structures 2007; 85:10971113.

[7] Bathe KJ, Zhang H. Finite element developments for general fluid flows with structural interactions. International Journal for Numerical Methods in Engineering 2004; 60:213-232.

[8] Bathe KJ, Ledezma G. Benchmark problems for incompressible fluid flows with structural interactions. Computers and Structures 2007; 85:628-644.

[9] Hübner B, Walhorn E, Dinkler D. A monolithic approach to fluid-structure interaction using space-time finite elements. Computer Methods in Applied 
Mechanics and Engineering 2004; 193:2087-2104.

[10] Heil M. An efficient solver for the fully coupled solution of large-displacement fluid-structure interaction problems. Computer Methods in Applied Mechanics and Engineering 2004; 193:1-23.

[11] Thomson S, Mongeau L, Frankel S. Flow over a membrane-covered, fluid-filled cavity. Computers and Structures 2007; 85:1012-1019.

[12] Piperno S, Farhat C. Partitioned procedures for the transient solution of coupled aeroelastic problems - part ii: energy transfer analysis and threedimensional applications. Computer Methods in Applied Mechanics and Engineering 2001; 190:3147-3170.

[13] Cirak F, Deiterding R, Mauch S. Large-scale fluid-structure interaction simulation of viscoplastic and fracturing thin-shells subjected to shocks and detonations. Computers and Structures 2007; 85:1049-1065.

[14] Zuijlen Av, Bosscher S, Bijl H. Two level algorithms for partitioned fluidstructure interaction computations. Computer Methods in Applied Mechanics and Engineering 2007; 196:1458-1470.

[15] Fernandez M, Moubachir M. A newton method using exact jacobians for solving fluid-structure coupling. Computers and Structures 2005; 83:127-142.

[16] Michler C, van Brummelen E, de Borst R. An interface newton-krylov solver for fluid-structure interaction. International Journal for Numerical Methods in Fluids 2005; 47(10-11):1189-1195.

[17] Matthies H, Steindorf J. Partitioned but strongly coupled iteration schemes for nonlinear fluid-structure interaction. Computers and Structures 2002; 80(2730):1991-1999.

[18] Matthies H, Steindorf J. Partitioned strong coupling algorithms for fluidstructure interaction. Computers and Structures 2003; 81:805-812. 
[19] Gerbeau JF, Vidrascu M. A quasi-newton algorithm based on a reduced model for fluid-structure interaction problems in blood flows. ESAIM: Mathematical Modelling and Numerical Analysis 2003; 37(4):631-648.

[20] Causin P, Gerbeau JF, Nobile F. Added-mass effect in the design of partitioned algorithms for fluid-structure problems. Computer Methods in Applied Mechanics and Engineering 2005; 194(42-44):4506-4527.

[21] Vierendeels J, Dumont K, Dick E, Verdonck P. Analysis and stabilization of fluid-structure interaction algorithm for rigid-body motion. AIAA Journal 2005; 43(12):2549-2557.

[22] Riemslagh K, Vierendeels J, Dick E. An efficient coupling procedure for flexible wall fluid-structure interaction. In: European Congress on Computational Methods in Applied Sciences and Engineering. Barcelona, Spain: ECCOMAS, 200013.

[23] Degroote J, Vierendeels J, Dick E. Numerical simulation of bubble and droplet dynamics using partitioned solvers. In: Wesseling P, Oñate E, Périaux J, editors, European Conference on Computational Fluid Dynamics. Egmond aan Zee, The Netherlands: ECCOMAS, 2006 1-17.

[24] Mok D, Wall W. Partitioned analysis schemes for the transient interaction of incompressible flows and nonlinear flexible structures. In: Schweizerhof K, Wall K WA Bletzinger, editors, Trends in computational structural mechanics. Barcelona, Spain: CIMNE, 2001 1-10.

[25] Küttler U, Wall W. Fixed-point fluid-structure interaction solvers with dynamic relaxation Computational Mechanics 2008; DOI: 10.1007/s00466-008-0255-5.

[26] Mok D, Wall W, Ramm E. Accelerated iterative substructuring schemes for instationary fluid-structure interaction. In: Bathe KJ, editor, First MIT 
Conference on Computational Fluid and Solid Mechanics. Cambridge, MA, USA: Elsevier, 2001 1325-1328.

[27] Manno V, Reitsma S. Developing numerical techniques for solving low mach number fluid-acoustic problems. AIAA Journal 1993; 31(11):1984-1991.

[28] Vierendeels J, Riemslagh K, Dick E, Verdonck P. Computer simulation of intraventricular flow and pressure gradients during diastole. Journal of Biomechanical Engineering 2000; 122:667-674.

[29] Riemslagh K, Vierendeels J, Dick, E. Coupling of a Navier-Stokes solver and an elastic boundary solver for unsteady problems. In: Papailiou K, Tsahalis D, Périaux J, Hirsch C, Pandolfi M, editors, European Conference on Computational Fluid Dynamics. Athens, Greece: ECCOMAS, 1998 1040-1045.

[30] Förster C, Wall W, Ramm E. The artificial added mass effect in sequential staggered fluid-structure interaction algorithms. In: Wesseling P, Oñate E, Périaux J, editors, European Conference on Computational Fluid Dynamics. Egmond aan Zee, The Netherlands: ECCOMAS, 2006 1-20. 


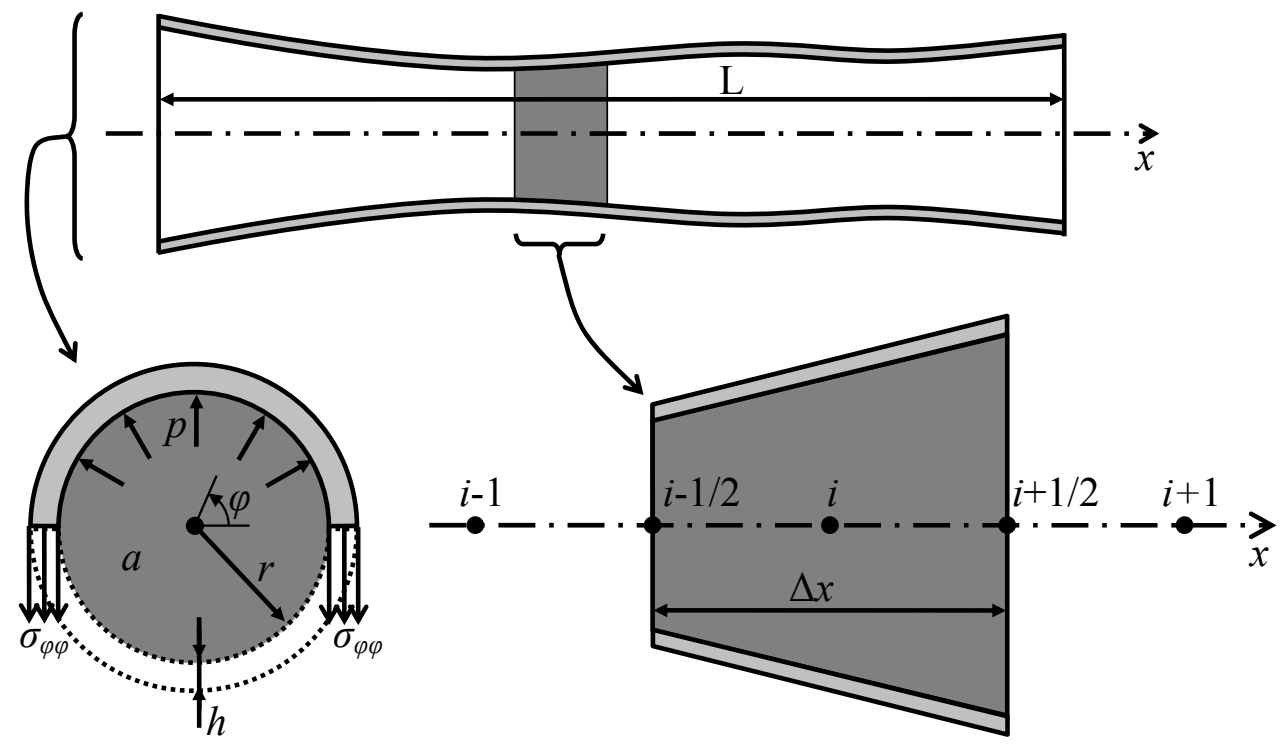

Fig. 1. The geometry of the flexible tube with details of the cross section and a control volume used in the discretization of the flow equations. 


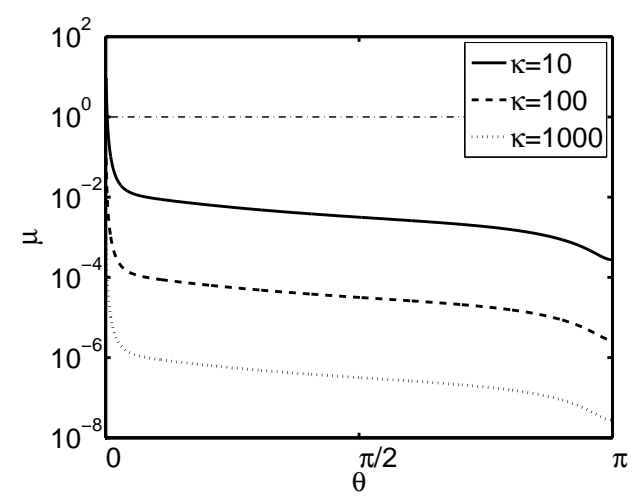

(a)

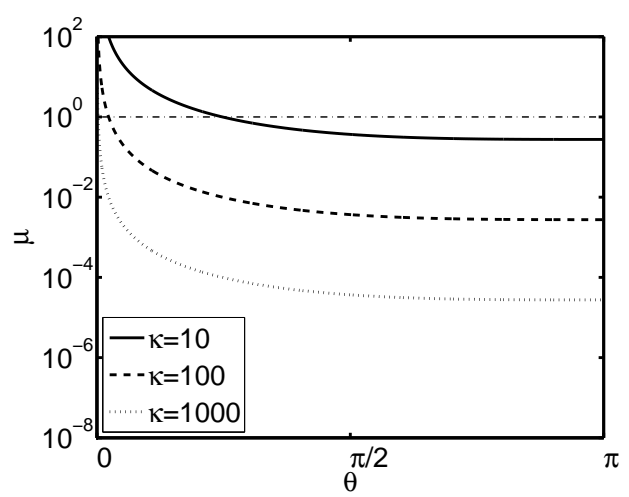

(b)

Fig. 2. Error amplification with constant $\tau N$, variable $\kappa$ and coupling parameters $\gamma_{1}=\gamma_{2}=\gamma_{3}=\gamma_{4}=0$, so a completely partitioned simulation. (a) $\tau N=10$; (b) $\tau N=0.1$. 


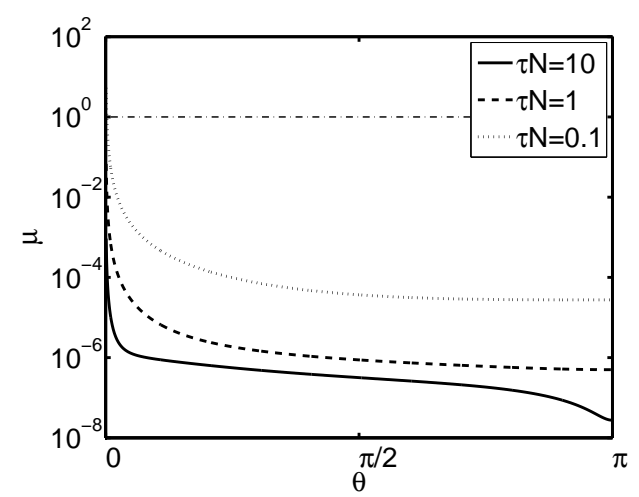

(a)

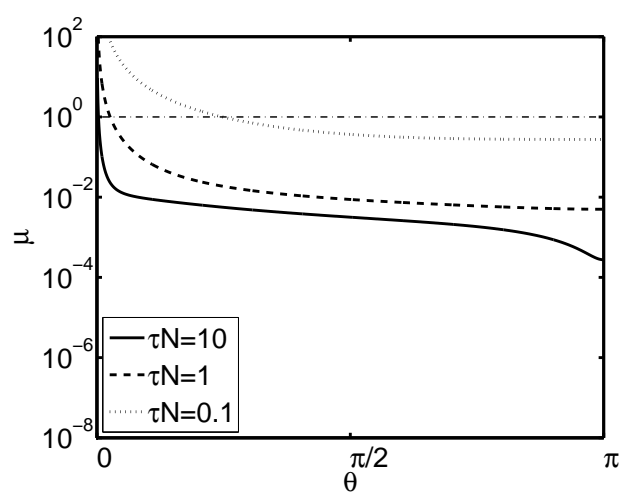

(b)

Fig. 3. Error amplification with constant $\kappa$, variable $\tau N$ and coupling parameters $\gamma_{1}=\gamma_{2}=\gamma_{3}=\gamma_{4}=0$, so a completely partitioned simulation. (a) $\kappa=1000$; (b) $\kappa=10$. 


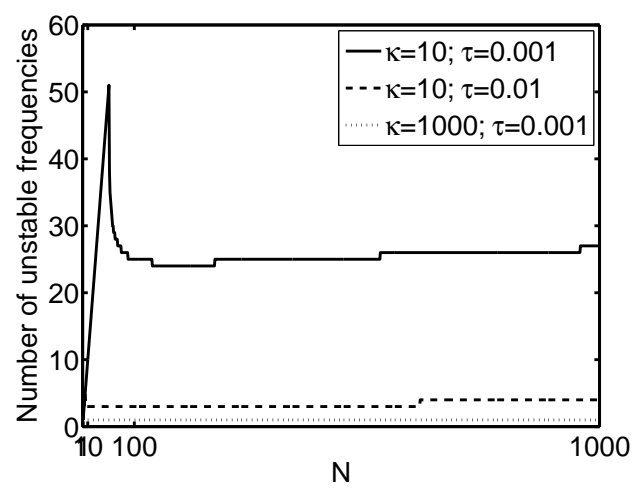

Fig. 4. Number of unstable frequencies as a function of the number of cells $N$ for $\gamma_{1}=\gamma_{2}=\gamma_{3}=\gamma_{4}=0$ and different values of $\kappa$ and $\tau$. 


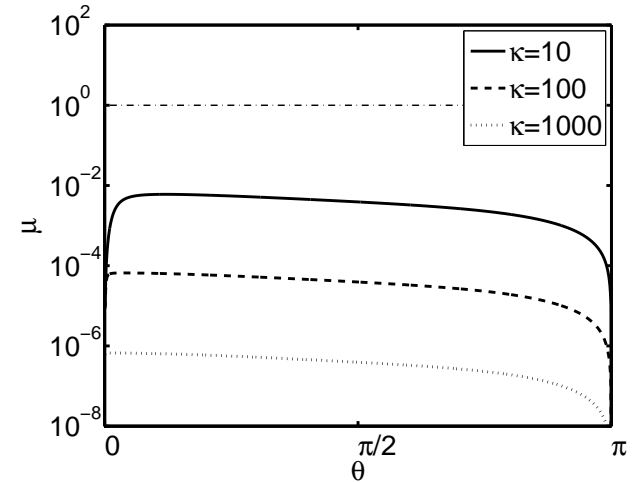

(a)

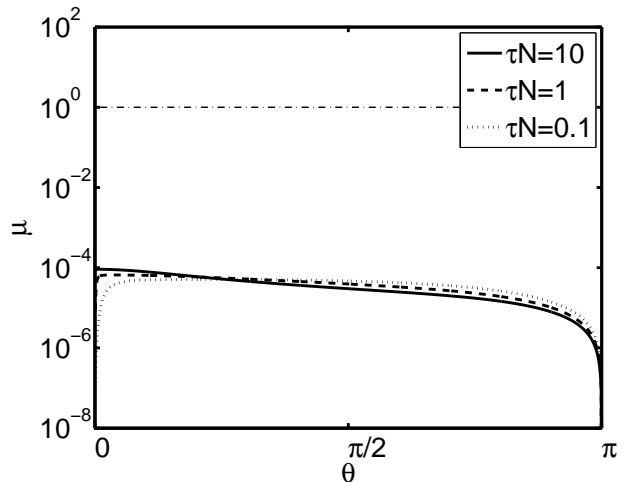

(b)

Fig. 5. Error amplification with coupling parameters $\gamma_{1}=1, \gamma_{2}=\gamma_{3}=\gamma_{4}=0$.

(a) $\tau N=1$. Although $\tau N$ is an order of magnitude smaller than in Figure 2(a), $\gamma_{1}=1$ stabilizes the coupling and the curves are approximately at the same height; (b) $\kappa=100$. The error amplification factor remains nearly constant when $\tau N$ is decreased, as opposed to Figure 3 . 


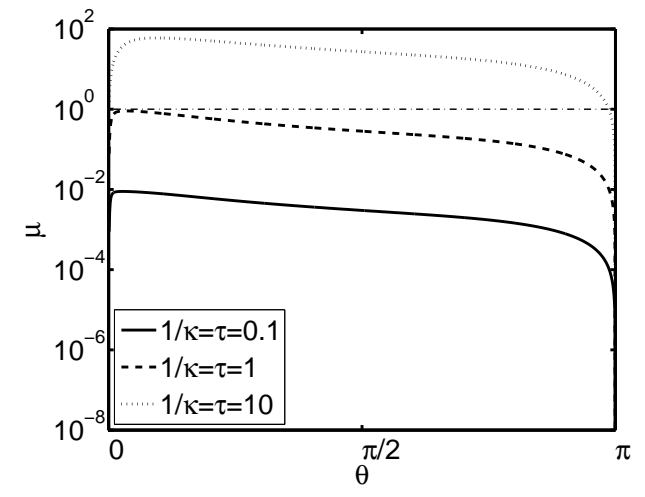

(a)

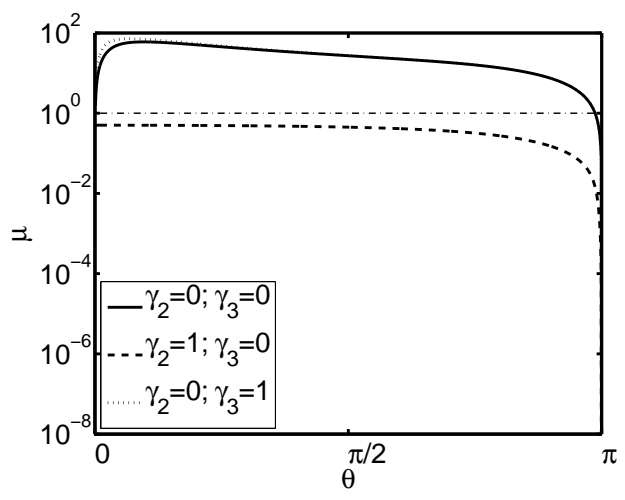

(b)

Fig. 6. Error amplification with coupling parameters $\gamma_{1}=1, \gamma_{4}=0$ and $N=100$.

(a) $\gamma_{2}=\gamma_{3}=0$; (b) $1 / \kappa=\tau=10$. 


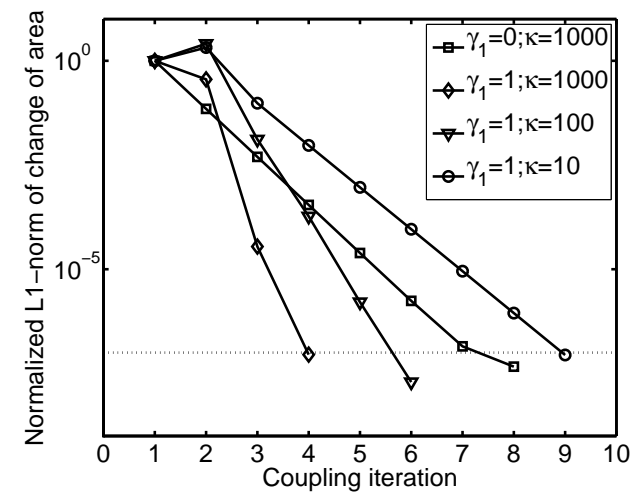

(a)

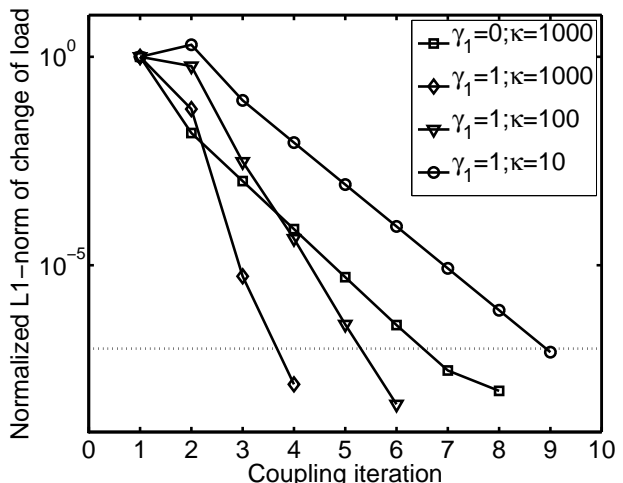

(b)

Fig. 7. Convergence behaviour during the coupling iterations in a representative time step if fixed point iterative coupling is used with $\gamma_{2}=\gamma_{3}=\gamma_{4}=0, \tau=0.01$ and $N=100$. The convergence criterion $1 \mathrm{E}-7$ is indicated with a dotted line and the residual is normalized with its initial value. When $\kappa=1000$, the artificial compressibility term $\left(\gamma_{1}=1\right)$ accelerates convergence but it is not required. For $\kappa=100$ or 10, the artificial compressibility term is necessary to obtain convergence. (a) Normalized L1-norm of the change in cross sectional area; (b) Normalized L1-norm of the change in fluid load. 


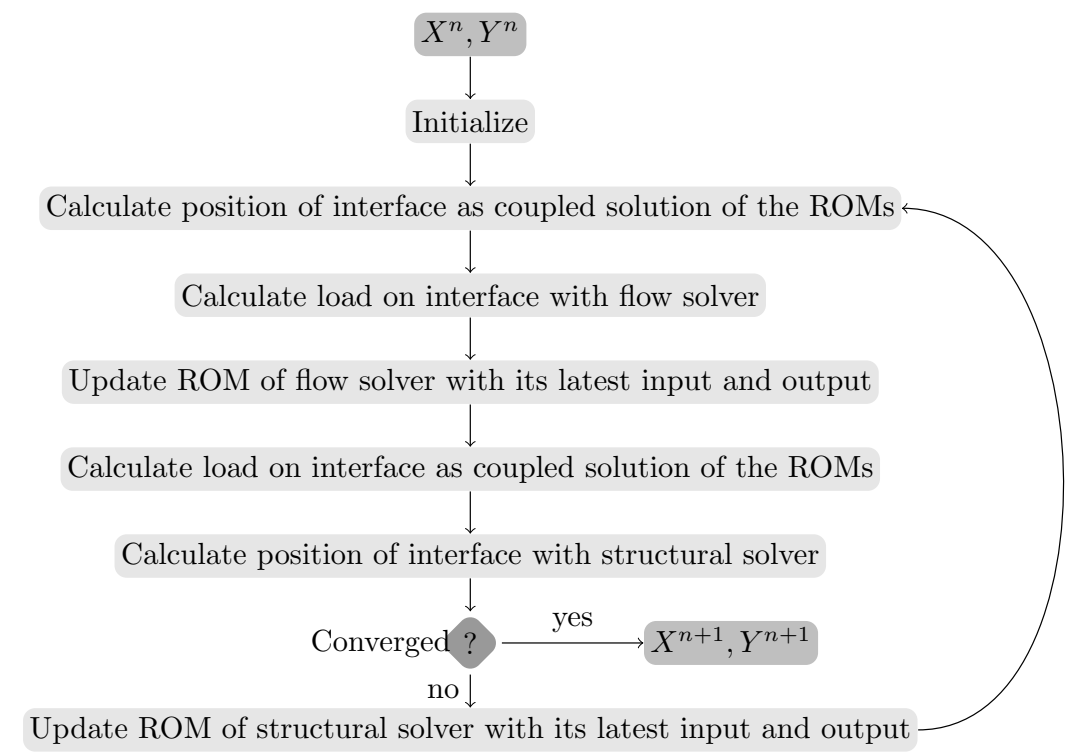

Fig. 8. Coupling scheme with reduced-order models. The initialization comprises steps 1 to 10 from the algorithm in appendix A while the actions in the loop correspond with steps 11 to 17 . 


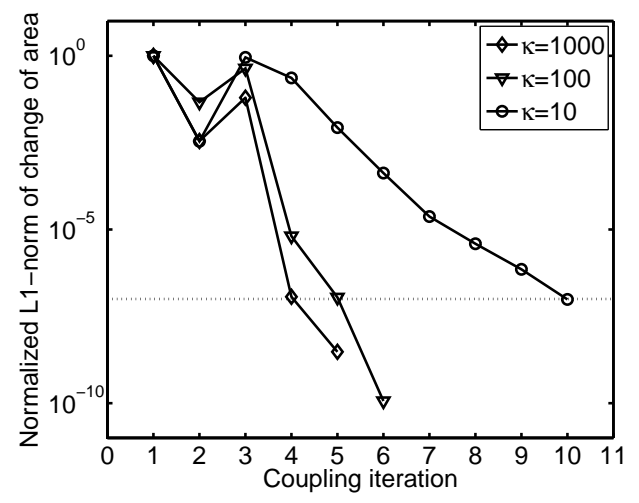

(a)

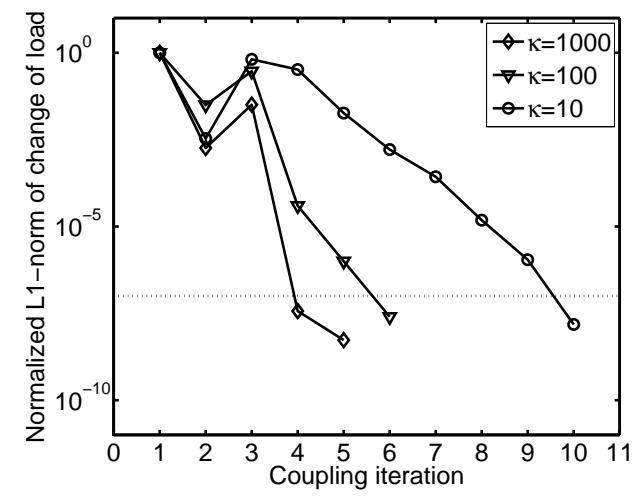

(b)

Fig. 9. Convergence behaviour during the coupling iterations in a representative time step if reduced-order model coupling is used with $\tau=0.01$ and $N=100$. The convergence criterion 1E-7 is indicated with a dotted line and the residual is normalized with its initial value. (a) Normalized L1-norm of the change in cross sectional area; (b) Normalized L1-norm of the change in fluid load. 


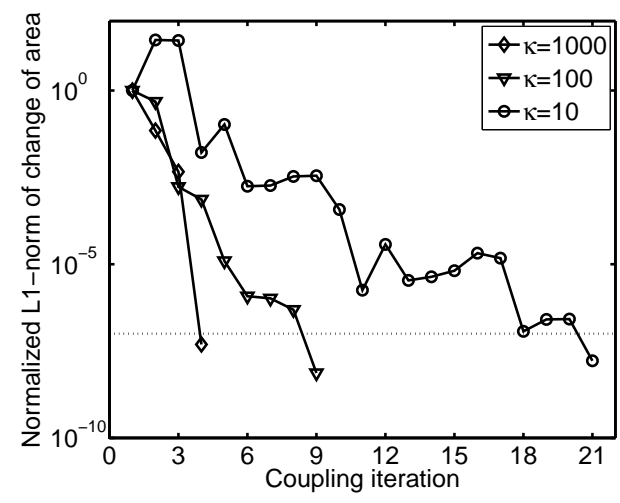

(a)

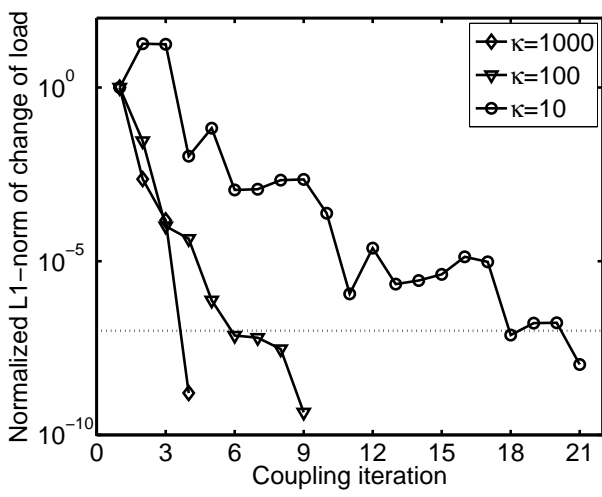

(b)

Fig. 10. Convergence behaviour during the coupling iterations in a representative time step if Aitken underrelaxation is used with $\tau=0.01$ and $N=100$. The convergence criterion 1E-7 is indicated with a dotted line and the residual is normalized with its initial value. (a) Normalized L1-norm of the change in cross sectional area; (b) Normalized L1-norm of the change in fluid load. 


\section{Tables}

\section{Table 1}

\begin{tabular}{|c|c|c|c|c|}
\hline$N=100$ & $\gamma_{1}=0$ & \multicolumn{3}{|c|}{$\gamma_{1}=1$} \\
\hline$\tau \downarrow \quad \kappa \rightarrow$ & 1000 & 1000 & 100 & 10 \\
\hline 0.1 & 5.00 & 3.80 & 4.90 & 8.90 \\
\hline 0.01 & 7.88 & 4.50 & 5.99 & 9.71 \\
\hline 0.001 & 86.71 & 5.08 & 6.00 & 9.96 \\
\hline$N=1000$ & $\gamma_{1}=0$ & \multicolumn{3}{|c|}{$\gamma_{1}=1$} \\
\hline$\kappa \rightarrow$ & 1000 & 1000 & 100 & 10 \\
\hline 0.1 & 5.00 & 3.80 & 4.90 & 8.90 \\
\hline 0.01 & 8.36 & 4.46 & 5.99 & 9.60 \\
\hline 0.001 & 158.92 & 5.05 & 6.00 & 9.67 \\
\hline
\end{tabular}

Number of coupling iterations per time step averaged over one period of the inlet boundary condition for different values of $\kappa, \tau$ and $N$ if fixed point iterative coupling with $\left(\gamma_{1}=1\right)$ and without $\left(\gamma_{1}=0\right)$ the artificial compressibility coupling term is used. In all cases $\gamma_{2}=\gamma_{3}=\gamma_{4}=0$ and the structure is modeled with the linear constitutive equation (17). 


\begin{tabular}{|c|c|c|c|c|c|c|c|c|c|}
\hline$N=100$ & \multicolumn{3}{|c|}{$\mathrm{ROM}$} & \multicolumn{3}{|c|}{ Aitken } & \multicolumn{3}{|c|}{$\gamma_{1}=1$} \\
\hline$\tau \downarrow \quad \kappa \rightarrow$ & 1000 & 100 & 10 & 1000 & 100 & 10 & 1000 & 100 & 10 \\
\hline 0.1 & 4.00 & 5.00 & 7.10 & 4.00 & 5.00 & 10.10 & 3.80 & 4.90 & 9.10 \\
\hline 0.01 & 4.89 & 5.90 & 10.74 & 4.07 & 8.84 & 21.22 & 4.49 & 5.99 & 9.67 \\
\hline 0.001 & 7.33 & 9.64 & 24.77 & 10.41 & 20.64 & 58.08 & 5.01 & 6.00 & 9.97 \\
\hline$N=1000$ & \multicolumn{3}{|c|}{$\mathrm{ROM}$} & \multicolumn{3}{|c|}{ Aitken } & \multicolumn{3}{|c|}{$\gamma_{1}=1$} \\
\hline$\kappa \rightarrow$ & 1000 & 100 & 10 & 1000 & 100 & 10 & 1000 & 100 & 10 \\
\hline 0.1 & 4.00 & 5.00 & 7.10 & 4.00 & 5.00 & 10.40 & 3.80 & 4.90 & 8.90 \\
\hline 0.01 & 4.74 & 6.06 & 11.52 & 4.90 & 8.97 & 25.80 & 4.51 & 5.99 & 9.61 \\
\hline 0.001 & 6.87 & 9.14 & 25.13 & 10.52 & 21.67 & 55.34 & 5.01 & 6.00 & 9.65 \\
\hline
\end{tabular}

Table 2

Number of coupling iterations per time step averaged over one period of the inlet boundary condition for different values of $\kappa, \tau$ and $N$ if coupling with reducedorder models, Aitken underrelaxation or fixed point iterative coupling with artificial compressibility coupling term $\left(\gamma_{1}=1\right)$ are used. The coupling parameters are $\gamma_{2}=$ $\gamma_{3}=\gamma_{4}=0$ and $\gamma_{1}=0$ except when indicated otherwise. The structure is modeled with the non-linear constitutive equation (16). 